\title{
Single- and two-color attosecond hard $x$-ray free-electron laser pulses with nonlinear compression
}

\author{
Alexander Malyzhenkov $\odot,{ }^{1, *}$ Yunieski P. Arbelo,,${ }^{1}$ Paolo Craievich $\odot,{ }^{1}$ Philipp Dijkstal $\odot,{ }^{1,2}$ Eugenio Ferrari, ${ }^{1}$ Sven Reiche, ${ }^{1}$ \\ Thomas Schietinger $\odot,{ }^{1}$ Pavle Juranić, ${ }^{1}$ and Eduard Prat ${ }^{1, \dagger}$ \\ ${ }^{1}$ Paul Scherrer Institut, $\mathrm{CH}-5232$ Villigen PSI, Switzerland \\ ${ }^{2}$ Department of Physics, ETH Zürich, CH-8092 Zürich, Switzerland
}

(Received 27 July 2020; revised 11 September 2020; accepted 15 October 2020; published 26 October 2020)

\begin{abstract}
We report on the generation of one- and two-color attosecond hard x-ray pulses at SwissFEL by appropriately tailoring the nonlinear compression of the electron beam. For achieving very short [about 300 attoseconds (as)] and stable single-color pulses, we minimize the collective effects of the longitudinal space-charge forces by strongly compressing the electron beam right before the undulator, utilizing the energy collimator chicane as a third compression stage. For achieving two-color short pulses, we exploit enhanced space-charge forces splitting the beam into two energy components.
\end{abstract}

DOI: 10.1103/PhysRevResearch.2.042018

X-ray free-electron lasers (XFELs) are unique instruments capable of producing transversely coherent radiation [1-4] with applications in multiple scientific fields [4-6]. Standard XFELs, operating in the self-amplified spontaneous emission (SASE) regime, produce radiation with a characteristic multimodal structure in time and frequency domains, with limited longitudinal coherence and a total pulse duration equivalent to the one of the driving electron beam.

The duration of a single radiation mode sets a lower limit on the pulse duration. The coherence length strongly correlates with the XFEL cooperation length [7], with typical values of a few hundred attoseconds (as) for x rays. The production of single-mode XFEL pulses will allow the observation of atomic and molecular processes occurring at the same attosecond timescale [8]. It will be beneficial for coherent diffractive imaging $[9,10]$ and timing of ultrafast processes, while outrunning the loss of structural information due to radiation damage [11].

There is a strong scientific interest in producing not only one but multiple short XFEL pulses with different wavelengths, enabling, for instance, $\mathrm{x}$-ray-pump-x-ray-probe experiments [12-14], more precise timing of radiation damage by varying the time delay between two sub-fs pulses (one initiating the damage and another probing it), coherent stimulated x-ray Raman spectroscopy [15-18], or multiwavelength anomalous diffraction [19].

Creating shorter $\mathrm{x}$-ray pulses containing fewer modes is feasible by reducing the temporal duration of the electron beam suitable for XFEL emission. Conceptual approaches are strong compression of a low-charge electron bunch [20,21],

\footnotetext{
*alexander.malyzhenkov@psi.ch

†eduard.prat@psi.ch

Published by the American Physical Society under the terms of the Creative Commons Attribution 4.0 International license. Further distribution of this work must maintain attribution to the author(s) and the published article's title, journal citation, and DOI.
}

spoiling the majority of the beam with a slotted foil [22,23], locally increasing the energy spread with a laser heater [24], or exciting betatron oscillations in the undulator section [25-27], or, alternatively, by local enhancement of the peak current of the electron beam. The latter is possible with nonlinear compression, where higher-order transport elements $\left(T_{566}, T_{655}\right)$ dominate the profile $[28,29]$. Nonlinear compression is much less sensitive to radio-frequency (rf) phase jitters compared to linear compression. Alternatively, a high peak current can be achieved by using a wiggler in conjunction with an external laser or self-modulation, and a magnetic chicane in the so-called enhanced SASE (ESASE) scheme [30-32]. The wiggler-based method demonstrated single-mode and even two-color single-mode attosecond pulses for soft $\mathrm{x}$ rays. Methods to produce two-color SASE FEL pulses employ two undulator sections tuned at different wavelengths [33-37], or one undulator section with two lasing parts of the same electron bunch at different energies [38-44].

In this Rapid Communication, we present two major advances in the generation of ultrashort XFEL pulses. First, we demonstrate the generation of single-mode hard x-ray pulses based on nonlinear compression of the electron beam right before the undulator section, resulting in an enhanced pulse stability compared to previously reported results. Second, we establish a method to produce attosecond two-color hard x-ray pulses, which exploits longitudinal space-charge forces acting between the compression and emission of radiation in the undulator.

The effects of longitudinal space-charge forces are central to both these developments. These forces are an inevitable consequence of short electron bunches needed in XFELs to achieve the peak currents required for lasing. XFELs typically compress electron bunches longitudinally in two bunch compression chicanes. The parameters driving the compression are the beam's energy chirp, defined as $h=d \delta / d z$, where $\delta=$ $(E-\langle E\rangle) /\langle E\rangle$ is the relative deviation of a particle's energy $E$ from the mean energy $\langle E\rangle$ and $z$ the particle position along the bunch ( $z>0$ is in front of the bunch), and the longitudinal dispersion $R_{56}$ of the magnetic chicane (see, e.g., Ref. [45]). 


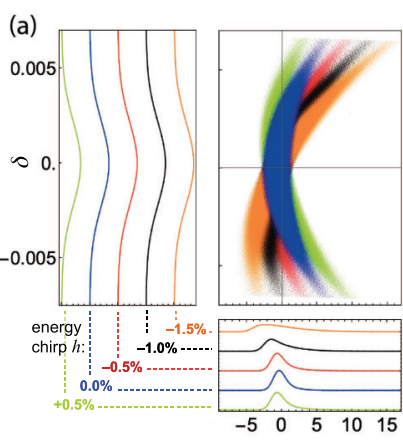

$t$ (fs)

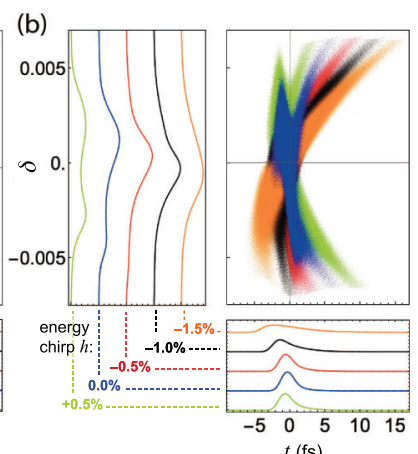

$t(\mathrm{fs})$
FIG. 1. Simulated longitudinal phase space of the electron beam after nonlinear single-stage compression with linear $\left(R_{56}=20 \mathrm{~mm}\right)$ and second-order $\left(T_{566}=23 \mathrm{~mm}\right)$ longitudinal dispersions: (a) After a chicane; (b) after a chicane and a 300-m-long free-space propagation affected by longitudinal space charge. The blue distribution corresponds to an energy chirp of $h=-50 \mathrm{~m}^{-1}$ before the chicane, fulfilling the full-compression condition $h R_{56}=-1$; the red/black/orange distributions are for $0.5 \% / 1 \% / 1.5 \%$ smaller chirp values, corresponding to undercompression; the green distribution is for $0.5 \%$ larger chirp corresponding to overcompression.

The energy chirp is usually generated through off-crest acceleration in the rf structures upstream of the compression chicanes.

Obviously, any jitter in the accelerating rf phases will, via the energy chirp, directly impact the compression process and hence the stability of the XFEL pulses. Moreover, the deteriorating effect of the $\mathrm{rf}$ jitter is exacerbated by longitudinal space-charge forces acting along the beam transport between final compression and exploitation of the beam as a source of XFEL radiation.

Figure 1 illustrates these effects by comparing longitudinal phase spaces of electrons simulated with ELEGANT [46], immediately after nonlinear compression [Fig. 1(a)] and after an additional $300 \mathrm{~m}$ of transport [Fig. 1(b)], as well as their susceptibilities to variations in the energy chirp of the incoming beam. The associated projections onto the energy axis clearly indicate that having the electron beam lase immediately after the final compression stage leads to a much more stable beam in terms of energy content. The robustness of the energy profile against different compression configurations in the case of minimized longitudinal space-charge effects in turn provides the freedom to select the configuration that best minimizes the pulse duration, without compromising the energy distribution within the beam.

Closer inspection of the energy profiles in Fig. 1(b) also reveals a potentially beneficial effect of the longitudinal space-charge forces: In the cases of full compression and overcompression they give rise to a bimodal energy distribution centered around two distinct energies. In the XFEL, this feature may be harnessed to generate two-color photon pulses with uncorrelated intensities in a single undulator section.

In the following we give experimental proof for both of these strategies to exploit the compression dynamics including longitudinal space charge: ultrashort pulses with enhanced stability and ultrashort two-color pulses.

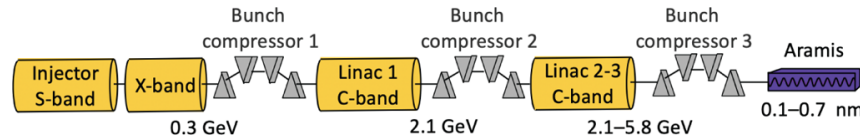

FIG. 2. Layout of the hard $\mathrm{x}$-ray beamline Aramis at SwissFEL (not to scale).

Our results have been obtained at SwissFEL, the XFEL facility that recently started user operation at the Paul Scherrer Institute in Switzerland. The layout of SwissFEL's hard X-ray beamline Aramis is sketched in Fig. 2. The standard operation mode, using a 200-pC bunch for relatively long pulses ( $\sim 30 \mathrm{fs}$ rms) and a 10-pC bunch for shorter pulses (a few fs rms), relies on two-stage compression with two magnetic chicanes called bunch compressors 1 and 2 ( $\mathrm{BC} 1$ and $\mathrm{BC} 2)$. The required energy chirp is imposed via rf off-crest acceleration in the injector linac and linac 1. A higher-harmonic decelerating cavity ( $X$ band) compensates for rf curvature from the sinusoidal acceleration field and for dispersion nonlinearities in the chicanes. A third magnetic chicane is normally set up in an isochronous mode by means of quadrupole and sextupole magnets (not shown) for energy collimation before the undulator entrance, but it can operate as a third bunch compressor (BC3).

Transverse deflecting cavities enable longitudinal phasespace measurements after both BC1 and BC2 [47]. Direct photon pulse duration measurements are currently unavailable at SwissFEL but the pulse length can be deduced from the analysis of single-shot spectra [48]. X-ray spectra are acquired with a single-shot spectrometer $[49,50]$. More information about SwissFEL can be found in Refs. [51-54].

In our first setup, in which we aim at minimizing longitudinal space-charge forces, we strongly compress the electron beam only in $\mathrm{BC} 3$, the last chicane right before the undulator section. As explained above, this approach is expected to yield better stability against machine and bunch jitter, resulting in shorter pulses in practice. Accomplishing compression in three instead of two stages generally allows for smaller chicane dispersions $\left(R_{56}\right)$ and energy chirps, which in turn reduces coherent synchrotron radiation effects and improves the performance sensitivity to jitters [55].

In linear approximation, full compression after the third bunch compression stage (BC3) is achieved for $1+$ $R_{56(3)} h_{3}=0$, where $R_{56(3)}$ is the longitudinal dispersion of $\mathrm{BC} 3$ and $h_{3}$ is the associated incoming energy chirp. Taking into consideration the first two compression stages, BC1 and $\mathrm{BC} 2$, operated in a normal (i.e., undercompression) configuration, the final bunch length for full compression in BC3 is given by

$$
\sigma_{z, f}=c \frac{E_{i}}{E_{f}} R_{56(3)} \sigma_{\delta},
$$

where $\sigma_{\delta}$ is the uncorrelated energy spread before $\mathrm{BC} 1, E_{i, f}$ are the energies at $\mathrm{BC} 1$ and $\mathrm{BC} 3$, respectively, and $c=c_{1} c_{2}$ is the accumulated compression before $\mathrm{BC} 3$, where $c_{n}=(1+$ $\left.R_{56(n)} h_{n}\right)^{-1}$ are compression factors in the first and second stages $\left(c_{1,2}^{-1} \neq 0\right)$.

From Eq. (1) we see that a small dispersion in the last compression stage is essential to achieve short pulses. We use 
(a)

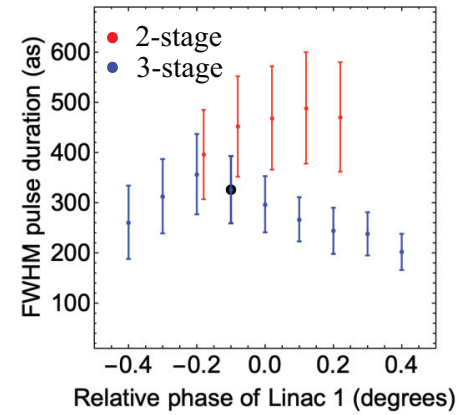

(c) 2-stage

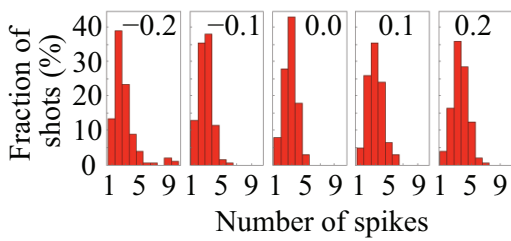

(b)

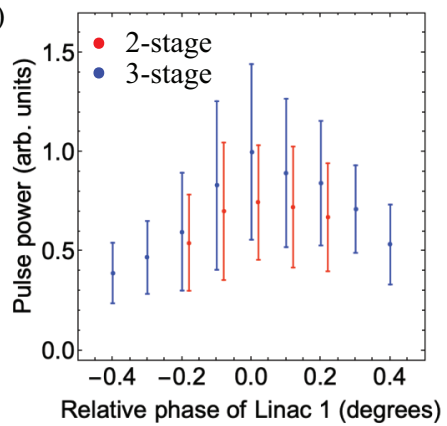

(d) 3-stage

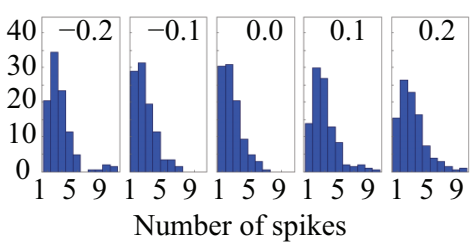

(e)

(f)

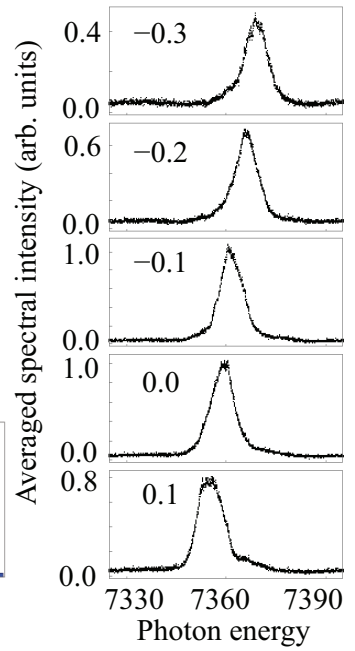

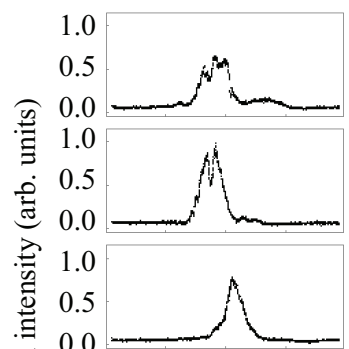

ज्ञ
के

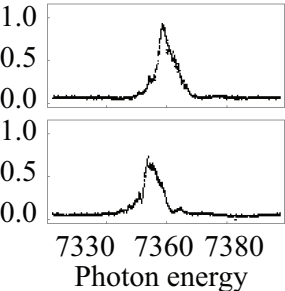

FIG. 3. Single-pulse generation with two- and three-stage compression: (a) Reconstructed minimum pulse duration; (b) reconstructed pulse power; (c), (d) histograms of the number of spikes; (e) averaged spectra of all single-spike shots for different linac-1 phase; (f) five consecutive shots for three-stage compression, at $-0.1^{\circ}$ relative linac- 1 phase. The relative zero phase of linac 1 corresponds to $65.5^{\circ}$ for the two-stage and $66.7^{\circ}$ for the three-stage configuration. The large black dot in (a) indicates the settings with negligible linear chirp.

BC3 as a dispersive chicane (with quadrupole and sextupole magnets turned off) giving $R_{56(3)}=3.4 \mathrm{~mm}$. This longitudinal dispersion requires an energy chirp of about $-300 \mathrm{~m}^{-1}$ to achieve full compression. For such a large chirp, the rf phase and amplitude jitters in linacs 2 and 3 (between BC2 and BC3) are insignificant. Since currently no diagnostics of the longitudinal phase space of the electron beam after compression in $\mathrm{BC} 3$ is available, we set up the three-stage compression scheme by measuring the energy chirp before $\mathrm{BC} 3$.

The initial electron bunch duration at the injector is $1.5 \mathrm{ps}$ (rms). The compression parameters are chosen to reach the desired energy chirp of around $-300 \mathrm{~m}^{-1}$. For the results presented below, we work with a two-stage compression factor $c \approx 46$ resulting in a bunch length of 32.5 fs before $\mathrm{BC} 3$. Tuning the final energy with linac 3 modifies the chirp and requires a reconfiguration of the stages before (namely linac 1) for compensation. The $X$-band cavity operates at a phase opposite to on-crest acceleration and with voltage set close to the available maximum (23 MV) to reduce second-order nonlinearities for best performance. The final energy chirp at BC3 can easily be tuned by changing either the linac-1 phase (for large steps), or the linac-3 phase (for smaller adjustments).

Figure 3 summarizes our results for attosecond singlepulse generation with three-stage compression and compares them to results obtained with a more conventional two-stage compression setup under otherwise equivalent conditions. The data were acquired during the same experimental shift with a photon energy of $7.36 \mathrm{keV}$ (similar results have been obtained for photon energies between 5 and $9 \mathrm{keV}$ ). For both configurations, we empirically optimize the undulator taper for best performance (i.e., maximum pulse energy and minimum pulse duration). The pulse duration, optimized by minimizing the number of modes in the spectrum, is extracted from spectral information [48]. The spectrum of each shot is fitted with a sum of a variable number of Gaussian peaks, from which a weighted average spike width is determined. The spike width in turn allows us to derive lower and upper limits of the pulse duration, corresponding, respectively, to the assumptions of no chirp and a linear chirp maximizing the pulse duration, and differing by a factor of $\sqrt{2}$ [29].

Figures 3(a) and 3(b) display the pulse duration and power as a function of linac-1 phase for the two configurations. Each point represents an average of 200 consecutive XFEL shots, with error bars corresponding to one standard deviation. The fluctuations are due to the intrinsic SASE fluctuations and the jitter in the electron beam. Clearly, shorter, more powerful, and more stable (in terms of pulse duration fluctuations) pulses are generated with three-stage compression minimizing longitudinal space-charge effects. Histograms of the number of spikes shown in Figs. 3(c) and 3(d) indicate that the fraction of single-spike shots is also larger for three-stage compression (up to 30\%). Figure 3(e) shows average spectra of single-spike shots for the three-stage compression case and for different phases of linac 1. Apart from the expected shift in photon energy due to the change in compression settings, we observe a change in asymmetry in the spectra. At lower phases a tail develops in the low-energy part of the spectra, and vice versa for higher phases, while for the reference phase the spectrum is almost symmetric, signaling the minimum of the energy chirp. This means that the derived pulse duration, $326 \pm 67$ as [full width at half maximum (FWHM)] (assuming no chirp), shown in Fig. 3(a) is correct for this phase, while values for other phases may be slightly underestimated due to the energy chirp contribution. Figure 3(f) gives an example of five consecutive single shots for three-stage compression at the optimum phase. The large fraction of single-spike shots suggests that the XFEL process avoids deep saturation, which normally develops more modes due to slippage. In the case of the optimum linac-1 phase we measure a pulse energy of $1.0 \pm 0.4 \mu \mathrm{J}$, and singling out only the single-spike pulses we 

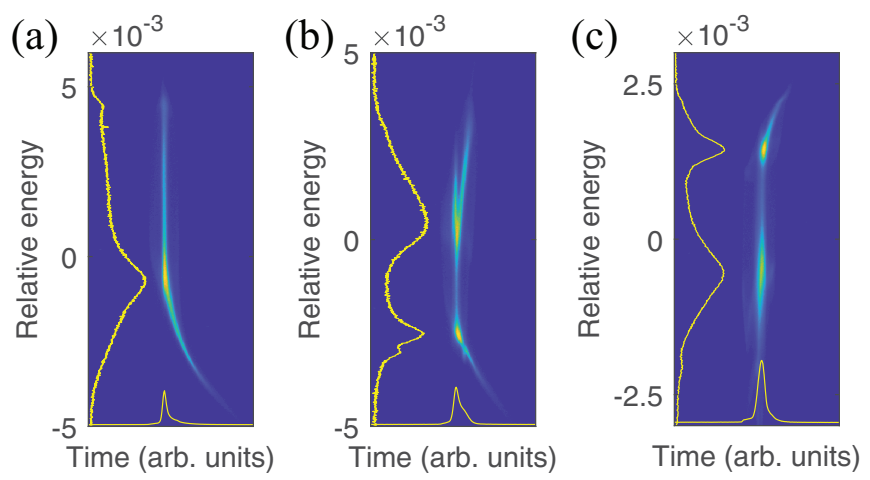

FIG. 4. Measured longitudinal phase space of the electron beam for nonlinear two-stage compression: (a) L shape at slight overcompression for single-color generation; (b) K shape at full compression for single-color radiation (or two-color in a split undulator); (c) I shape for two-color generation in a single undulator.

get $1.3 \pm 0.4 \mu \mathrm{J}$. The pulse energy measurements were made with an online gas ionization pulse energy monitor [56].

In contrast to the work presented so far, where we have tried to minimize the effects from longitudinal space-charge forces in the interest of XFEL pulse stability, in our second setup we aim to benefit from such effects. As explained above, longitudinal space-charge forces, if sufficiently strong, can split the beam into two parts featuring distinct energies, capable of lasing at different wavelengths (colors) in the same undulator section. For this to succeed, the two parts need to have similar local energy chirps to produce two XFEL pulses in the same undulator. We achieve this by an adjustment of the $\mathrm{BC} 1$ dispersion to minimize nonlinearities beyond what is possible within the $X$-band voltage limitations.

Figure 4 illustrates this by showing electron longitudinal phase-space distributions measured at the end of linac 3 for different two-stage compression settings. Figure 4(a) shows a condition corresponding to slight overcompression, suitable for single-pulse generation. Shifting the phase of linac 1 by $0.4^{\circ}$ results in a characteristic K shape [Fig. 4(b)], in which two distinct parts of the electron beam with different energies are present. These parts, however, feature different local energy chirps (i.e., opposite directions), therefore they cannot produce two-color XFEL pulses in one undulator section. Nevertheless, the two regions of the electron beam could produce two colors in two different undulator sections with tapers matching each of the local energy chirps and a delaying chicane [57]. This scheme would allow for a large tunability of the energy separation and the time delay between the two pulses. In Fig. 4(c) we additionally increase the BC1 dispersion $R_{56(1)}$ from 50.0 to $63.5 \mathrm{~mm}$, such that the two distinct energy parts of the electron beam end up having a similar local energy chirp. In this way, two pulses can be produced in a single undulator section. We note that the main features of the distributions in Fig. 4 are predicted by our simulations (see Fig. 1) as well as in earlier studies [28,29]. The generation of two independent pulses requires that the energy separation between the two parts of the electron beam be larger than the XFEL bandwidth acceptance. For the example shown in Fig. 4(c), the energy separation is around $0.2 \%$, more than sufficient to generate two distinct XFEL pulses.

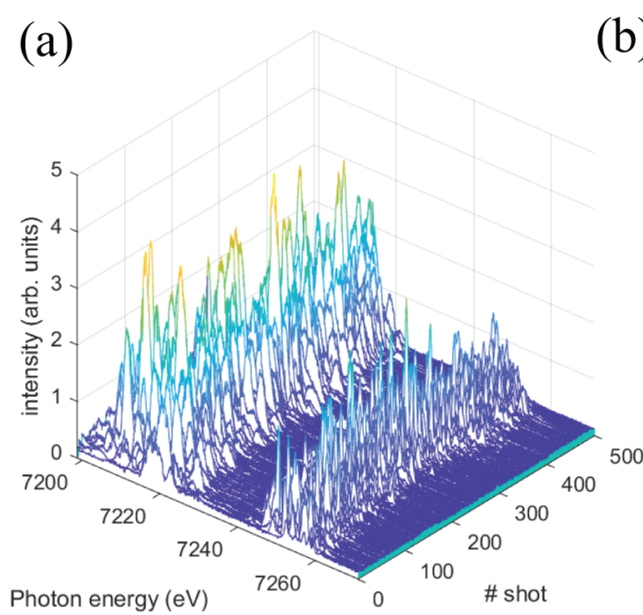

b)
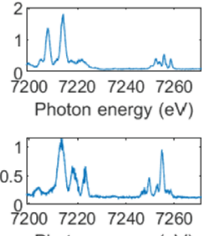
Photon energy (eV)

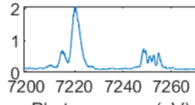
Photon energy (eV)

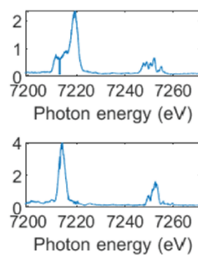

FIG. 5. Ultrashort two-color pulses: (a) Stack of 500 consecutive single-shot spectra; (b) five consecutive spectra shown separately for clarity.

In Fig. 5 we show 500 consecutive single-shot spectra obtained with the electron beam of Fig. 4(c), thereby demonstrating the generation of ultrashort two-color pulses following this approach. The mean photon energies are $E_{1}=$ $7215 \pm 2 \mathrm{eV}$ and $E_{2}=7250 \pm 2 \mathrm{eV}$, corresponding to $\sim 0.5 \%$ energy separation, qualitatively consistent with the longitudinal phase-space measurement. The pulse energies in the two colors are $1.3 \pm 0.4$ and $0.70 \pm 0.15 \mu \mathrm{J}$ (about $65 \%$ and $35 \%$ of the total pulse energy). Pulse duration and energy are uncorrelated between the two colors. The analysis of the spectra suggests that approximately $10 \%$ of the shots feature a single spike in at least one color and about $1 \%$ have a single spike in both colors simultaneously, while most of the shots exhibit a few-spike structure in each color [Fig. 5(b)]. The reconstructed FWHM pulse durations, derived under the assumption of no energy chirp, are $510 \pm 150$ and $920 \pm 180$ as for the two colors.

Instantaneous two-color x-ray pulses could be essential for pump-probe experiments on mixed-valence compounds enabling the simultaneous tracking of two ionic species after pumping [58]. For other experiments, a time delay between the two x-ray pulses could be controlled by adjusting the longitudinal dispersion of a magnetic chicane before the undulator beamline. At SwissFEL, the $R_{56}$ of $\mathrm{BC} 3$ can be adjusted between -0.3 and $+3.4 \mathrm{~mm}$ by means of quadrupole magnets [41]. The measured energy separation of $0.2 \%$ would allow time separations from -2 to +21 fs. The $R_{56}$ would change the duration of the two pulses: For our parameters, assuming that the individual pulses are fully compressed before $\mathrm{BC} 3$, the individual pulse durations would be subject to an increase at the $10 \%$ level for a time tunability of $4 \mathrm{fs}$. For larger tunability, as explained before, one could employ the beam demonstrated in Fig. 4(b) with the split undulator configuration [57].

To conclude, we have demonstrated the generation of single- and two-color attosecond pulses in the hard X-ray spectral range. Our approach goes beyond previously achieved results by demonstrating subfemtosecond two-color hard 
x-ray pulses and does not require any additional hardware beyond the standard configuration of an x-ray FEL facility. The generation of one- and two-color ultrashort hard x-ray pulses will be beneficial for many scientific applications, paving the way for outstanding progress in attosecond science.
We thank M. Huppert, A. Dax, A. Trisorio, S. Bettoni, F. Frei, H.-H. Braun, A. Cavalieri, A. Lutman, and G. Aeppli for support and fruitful discussions. The work of P.D. in the context of this project was supported by the Swiss National Science Foundation under Grant No. 200021_175498.
[1] J. Madey, J. Appl. Phys. 42, 1906 (1971).

[2] L. Elias, W. Fairbank, J. Madey, and T. Smith, Phys. Rev. Lett. 36, 717 (1976).

[3] D. A. G. Deacon, L. R. Elias, J. M. J. Madey, G. J. Ramian, H. A. Schwettman, and T. I. Smith, Phys. Rev. Lett. 38, 892 (1977).

[4] C. Pellegrini, A. Marinelli, and S. Reiche, Rev. Mod. Phys. 88, 015006 (2016).

[5] L. C. Johansson, B. Stauch, A. Ishchenko, and V. Cherezov, Trends Biochem. Sci. 42, 749 (2017).

[6] H. N. Chapman, Annu. Rev. Biochem. 88, 35 (2019).

[7] K. J. Kim, Z. Huang, and R. Lindberg, Synchrotron Radiation and Free-Electron Lasers: Principles of Coherent X-Ray Generation (Cambridge University Press, Cambridge, U.K., 2017).

[8] L. Young et al., J. Phys. B: At., Mol., Opt. Phys. 51, 3 (2018).

[9] H. N. Chapman et al., Nat. Phys. 2, 839 (2006).

[10] H. N. Chapman, C. Caleman, and N. Timneanu, Philos. Trans. R. Soc., B 369, 1 (2014).

[11] R. Neutze, R. Wouts, D. van der Spoel, E. Weckert, and J. Hajdu, Nature (London) 406, 752 (2000).

[12] S. Mukamel, D. Healion, Y. Zhang, and J. D. Biggs, Annu. Rev. Phys. Chem. 64, 101 (2013).

[13] I. Inoue et al., Proc. Natl. Acad. Sci. USA 113, 1492 (2016).

[14] W. Lu et al., Rev. Sci. Instrum. 89, 063121 (2018).

[15] I. V. Schweigert and S. Mukamel, Phys. Rev. A 76, 012504 (2007).

[16] U. Harbola and S. Mukamel, Phys. Rev. B 79, 085108 (2009).

[17] C. Weninger, M. Purvis, D. Ryan, R. A. London, J. D. Bozek, C. Bostedt, A. Graf, G. Brown, J. J. Rocca, and N. Rohringer, Phys. Rev. Lett. 111, 233902 (2013).

[18] A. Picón, J. Mompart, and S. H. Southworth, New J. Phys. 17, 083038 (2015).

[19] A. Gorelet et al., Nat. Commun. 8, 1170 (2017).

[20] S. Reiche, P. Musumeci, C. Pellegrini, and J. B. Rosenzweig, Nucl. Instrum. Methods Phys. Res., Sect. A 593, 45 (2008).

[21] J. B. Rosenzweig et al., Nucl. Instrum. Methods Phys. Res., Sect. A 593, 39 (2008).

[22] P. Emma, K. Bane, M. Cornacchia, Z. Huang, H. Schlarb, G. Stupakov, and D. Walz, Phys. Rev. Lett. 92, 074801 (2004).

[23] A. Marinelli, J. MacArthur, P. Emma, M. Guetg, C. Field, D. Kharakh, A. A. Lutman, Y. Ding, and Z. Huang, Appl. Phys. Lett. 111, 151101 (2017).

[24] A. Marinelli et al., Phys. Rev. Lett. 116, 254801 (2016).

[25] P. Emma and Z. Huang, Nucl. Instrum. Methods Phys. Res., Sect. A 528, 458 (2004).

[26] E. Prat, S. Bettoni, and S. Reiche, Nucl. Instrum. Methods Phys. Res., Sect. A 865, 1 (2017).

[27] M. W. Guetg, A. A. Lutman, Yuantao Ding, T. J. Maxwell, and Zhirong Huang, Phys. Rev. Lett. 120, 264802 (2018).
[28] S. Huang, Y. Ding, Z. Huang, and J. Qiang, Phys. Rev. ST Accel. Beams 17, 120703 (2014)

[29] S. Huang, Y. Ding, Y. Feng, E. Hemsing, Z. Huang, J. Krzywinski, A. A. Lutman, A. Marinelli, T. J. Maxwell, and D. Zhu, Phys. Rev. Lett. 119, 154801 (2017).

[30] A. A. Zholents, Phys. Rev. Accel. Beams 8, 040701 (2005).

[31] J. Duris et al., Nat. Photonics 14, 30 (2020).

[32] J. P. MacArthur, J. Duris, Z. Zhang, A. Lutman, A. Zholents, X. $\mathrm{Xu}, \mathrm{Z}$. Huang, and A. Marinelli, Phys. Rev. Lett. 123, 214801 (2019).

[33] D. A. Jaroszynski, R. Prazeres, F. Glotin, and J. M. Ortega, Phys. Rev. Lett. 72, 2387 (1994).

[34] A. A. Lutman, R. Coffee, Y. Ding, Z. Huang, J. Krzywinski, T. Maxwell, M. Messerschmidt, and H.-D. Nuhn, Phys. Rev. Lett. 110, 134801 (2013).

[35] T. Hara et al., Nat. Commun. 4, 2919 (2013).

[36] S. Reiche and E. Prat, J. Synchrotron Radiat. 23, 869 (2016).

[37] A. A. Lutman et al., Nat. Photonics 10, 745 (2016).

[38] A. Marinelli et al., Nat. Commun. 6, 6369 (2015).

[39] A. Marinelli et al., Twin-bunch two-color FEL at LCLS, in Proceedings of the 7th International Particle Accelerator Conference, IPAC 2016, Busan, Korea (JACoW, Geneva, 2016), p. 1032, TUZA02.

[40] Y. Ding et al., Appl. Phys. Lett. 107, 191104 (2015).

[41] Á. Saa Hernández, E. Prat, and S. Reiche, Phys. Rev. Accel. Beams 22, 030702 (2019).

[42] W. Qin, Y. Ding, A. A. Lutman, and Y.-C. Chao, Phys. Rev. Accel. Beams 20, 090701 (2017).

[43] P. Dijkstal, A. Malyzhenkov, S. Reiche, and E. Prat, Phys. Rev. Accel. Beams 23, 030703 (2020).

[44] S. Bettoni, E. Prat, and S. Reiche, Phys. Rev. Accel. Beams 19 050702 (2016).

[45] M. Dohlus, T. Limberg, and P. Emma, Electron bunch length compression, ICFA Beam Dynamics Newsletter No. 38, p. 15 (2005), http://www.icfa-bd.org/Newsletter38.pdf.

[46] M. Borland, ELEGANT: A flexible SDDS-compliant code for accelerator simulation, Advanced Photon Source Report No. LS-287, doi: 10.2172/761286 (2000).

[47] P. Craievich, R. Ischebeck, F. Löhl, G. L. Orlandi, and E. Prat, Transverse deflecting structures for bunch length and slice emittance measurements on SwissFEL, in Proceedings of FEL 2013, New York (JACoW, Geneva, 2013), Vol. 35, p. 236.

[48] Y. Inubushi et al., Phys. Rev. Lett. 109, 144801 (2012).

[49] M. Makita et al., Optica 2, 912 (2015).

[50] J. Rehanek et al., J. Instrum. 12, P05024 (2017).

[51] C. J. Milne et al., Appl. Sci. 7, 720 (2017).

[52] R. Ganter et al., SwissFEL Conceptual Design Report PSI Report 10-04 (Paul Scherrer Institut, 2012), https://www.psi.ch/ sites/default/files/import/swissfel_old/CurrentSwissFEL PublicationsEN/SwissFEL_CDR_V20_23.04.12_small.pdf. 
[53] E. Prat et al., Nat. Photon. (to be published).

[54] R. Abela et al., J. Synchrotron Radiat. 26, 1073 (2019).

[55] H.-S. Kang et al., J. Synchrotron Radiat. 26, 1127 (2019).

[56] P. Juranić et al., J. Synchrotron Radiat. 25, 1238 (2018).
[57] Z. Zhang, J. Duris, J. P. MacArthur, Z. Huang, and A. Marinelli, Phys. Rev. Accel. Beams 22, 050701 (2019).

[58] R. M. Martin, J. B. Boyce, J. W. Allen, and F. Holtzberg, Phys. Rev. Lett. 44, 1275 (1980). 